ARTICLE

Received 8 Aug 2014 | Accepted 11 Dec 2015 | Published 2 Feb 2016

DOI: $10.1038 /$ ncomms10448

OPEN

\title{
GWAS of 89,283 individuals identifies genetic variants associated with self-reporting of being a morning person
}

Youna Hu ${ }^{1, \dagger}$, Alena Shmygelska ${ }^{1}$, David Tran ${ }^{1,2}$, Nicholas Eriksson ${ }^{1}$, Joyce Y. Tung ${ }^{1} \&$ David A. Hinds ${ }^{1}$

Circadian rhythms are a nearly universal feature of living organisms and affect almost every biological process. Our innate preference for mornings or evenings is determined by the phase of our circadian rhythms. We conduct a genome-wide association analysis of selfreported morningness, followed by analyses of biological pathways and related phenotypes. We identify 15 significantly associated loci, including seven near established circadian genes (rs12736689 near RGS16, $P=7.0 \times 10^{-18} ;$ rs9479402 near $V I P, \quad P=3.9 \times 10^{-11}$; rs55694368 near PER2, $P=2.6 \times 10^{-9} ;$ rs35833281 near HCRTR2, $P=3.7 \times 10^{-9}$; rs11545787 near RASD1, $P=1.4 \times 10^{-8}$; rs11121022 near $P E R 3, P=2.0 \times 10^{-8}$; rs9565309 near $F B X L 3, P=3.5 \times 10^{-8}$. Circadian and phototransduction pathways are enriched in our results. Morningness is associated with insomnia and other sleep phenotypes; and is associated with body mass index and depression but we did not find evidence for a causal relationship in our Mendelian randomization analysis. Our findings reinforce current understanding of circadian biology and will guide future studies.

\footnotetext{
${ }^{1}$ 23andMe, Inc., 899 W Evelyn Avenue, Mountain View, California 94043 USA. ${ }^{2}$ Department of Biological Sciences, San Jose State University, San Jose, California 95112 USA. † Present address: A9.com Inc, 130 Lytton Avenue, Palo Alto, California 94301, USA. Correspondence and requests for materials should be addressed to Y.H. (email: youna.hu@gmail.com) or to D.A.H. (email: dhinds@23andme.com).
} 
A morning person prefers to rise and rest early, whereas a night person would choose a cycle later in the day. Chronobiology, the study of such differences (or chronotypes), began with Kleitman ${ }^{1}$ suggesting their existence and Horne and Ostberg $^{2}$ designing a questionnaire for their definition. Morningness is governed by a circadian rhythm mediated by the suprachiasmatic nucleus (SCN) in the hypothalamus. The SCN is a network of cellular oscillators that are synchronized in response to light input received from the human retina ${ }^{3}$. Differences in circadian rhythm have been associated with medically relevant traits such as sleep ${ }^{4}$, obesity ${ }^{5}$ and depression ${ }^{6}$.

Most genetic studies of circadian rhythm have been conducted on model organisms, beginning with the discovery of a first circadian clock gene per in Drosophila and CLOCK in mice (Supplementary Table 1). Human linkage studies have implicated PER2 in familial advanced sleep phase syndrome ${ }^{7}$ and candidate gene studies ${ }^{8,9}$ have found others. However, study sizes have been small and findings are not robust ${ }^{10}$. Furthermore, few genomewide association studies (GWAS) have been successful in identifying significant associations ${ }^{11-13}$.

We analysed genetic associations of self-reported morningness using the 23andMe cohort $(n=89,283)$ and identified a total of 15 genome-wide significant loci with seven of them close to wellestablished circadian genes such as PER2. We performed pathway analyses and found both circadian and phototransduction pathways enriched in our results. In addition, we observed significant associations between morningness and body mass index (BMI) and depression in our cohort but found no evidence to support a causal relationship in a Mendelian randomization (MR) analysis.

\section{Results}

Descriptions of GWAS study and cohort. We conducted a GWAS of self-reported morningness in the 23andMe participant cohort $^{14}$, across a total of $\sim 8$ million genotyped or imputed polymorphic sites. Morningness was defined by combining the highly concordant responses (Cohen's Kappa $=0.95$, $P<1.0 \times 10^{-200}$ ) to two web based survey questions that ask if the individual is naturally a morning or night person (Supplementary Table 2). Among 135,447 who answered at least one survey, $75.5 \%$ were scored as morning or night persons. Individuals who provided neutral $(n=32,842)$ or discordant responses $(n=309)$ were removed (Supplementary Table 9$)$. We did not find differences in age, gender or principal components (PCs; all $P>0.01$ ) when comparing individuals who provided discordant responses versus individuals who gave concordant responses $(n=12,442)$. We included individuals of European ancestry who had consented for research, and related individuals were removed from analysis (Methods section). Morningness is significantly associated with gender $\left(P=4.4 \times 10^{-77}\right)$, with a prevalence of $39.7 \%$ in males and $48.4 \%$ in females. Its prevalence increases with age $\left(P<1.0 \times 10^{-200}\right): 24.2 \%$ of those under 30 -years-old prefer mornings compared with $63.1 \%$ of those over 60 . This age trend is consistent with previous reported observations ${ }^{15}$.

Table 1 (together with Supplementary Table 2) shows the marginal association between morningness and other sleep phenotypes, BMI and depression (defined in Supplementary Table 3). Morning persons are significantly less likely to have insomnia (12.9 versus $18.3 \%$, odds ratio $(\mathrm{OR})=0.66, P=2.4$ $\left.\times 10^{-74}\right)$. They are also less likely to require $>8 \mathrm{~h}$ of sleep per day $\left(\mathrm{OR}=0.67, P=1.1 \times 10^{-72}\right)$, to sleep soundly $(\mathrm{OR}=0.74$, $\left.P=8.5 \times 10^{-50}\right)$, to sweat while sleeping $(\mathrm{OR}=0.8, P=1.0 \times$ $\left.10^{-23}\right)$ and to sleep walk $\left(\mathrm{OR}=0.77, P=4.7 \times 10^{-10}\right)$. Morningness is also associated with lower prevalence of depression
Table 1 | Demographic characteristics of the GWAS cohort.

\begin{tabular}{|c|c|c|c|}
\hline & $\begin{array}{c}\text { Morning } \\
\text { persons } \\
\text { N (\% of total) }\end{array}$ & $\begin{array}{c}\text { Evening } \\
\text { persons } \\
\text { N (\% of total) }\end{array}$ & $\begin{array}{l}\text { Proportion that } \\
\text { are morning } \\
\text { persons (\%) }\end{array}$ \\
\hline Total & $38,937(100.0)$ & $50,346(100.0)$ & 43.6 \\
\hline \multicolumn{4}{|l|}{ Sex } \\
\hline Male & $19,569(50.3)$ & $29,713(59.0)$ & 39.7 \\
\hline Female & $19,368(49.7)$ & $20,633(41.0)$ & 48.4 \\
\hline \multicolumn{4}{|l|}{ Age } \\
\hline$<30$ & $3,684(9.5)$ & $11,521(22.9)$ & 24.2 \\
\hline $30-45$ & $8,809(22.6)$ & $19,470(38.7)$ & 31.2 \\
\hline $45-60$ & $12,295(31.6)$ & $11,111(22.1)$ & 52.5 \\
\hline$>60$ & $14,149(36.3)$ & $8,244(16.4)$ & 63.1 \\
\hline \multicolumn{4}{|l|}{ Insomnia } \\
\hline No & $13,809(79.1)$ & $14,180(60.3)$ & 49.3 \\
\hline Yes & 3,639 (20.9) & $9,348(39.7)$ & 28.0 \\
\hline \multicolumn{4}{|l|}{ Sleep apnoea } \\
\hline No & $22,827(89.5)$ & $30,822(88.9)$ & 42.5 \\
\hline Yes & $2,673(10.5)$ & $3,862(11.1)$ & 40.9 \\
\hline \multicolumn{4}{|l|}{ Sleep needed } \\
\hline$<8 \mathrm{~h}$ & $7,549(56.6)$ & $8,715(46.4)$ & 46.4 \\
\hline$\geq 8 \mathrm{~h}$ & $5,782(43.4)$ & $10,068(53.6)$ & 36.4 \\
\hline \multicolumn{4}{|l|}{ Sound sleeper } \\
\hline No & $8,772(49.3)$ & $10,062(42.0)$ & 46.6 \\
\hline Yes & $9,020(50.7)$ & $13,901(58.0)$ & 39.4 \\
\hline \multicolumn{4}{|c|}{ Restless leg syndrome } \\
\hline No & $11,877(92.0)$ & $16,476(91.3)$ & 41.9 \\
\hline Yes & $1,035(8.0)$ & $1,566(8.7)$ & 39.8 \\
\hline \multicolumn{4}{|l|}{ Sweat while sleeping } \\
\hline No & $12,809(72.9)$ & $16,273(68.3)$ & 44.0 \\
\hline Yes & $4,765(27.1)$ & $7,546(31.7)$ & 38.7 \\
\hline \multicolumn{4}{|l|}{ Sleep walk } \\
\hline No & $12,145(92.9)$ & $16,773(90.9)$ & 42.0 \\
\hline Yes & $934(7.1)$ & 1,681 (9.1) & 35.7 \\
\hline \multicolumn{4}{|c|}{ Average daily sleep duration } \\
\hline$<8 \mathrm{~h}$ & $8,146(67.6)$ & $11,102(68.5)$ & 42.3 \\
\hline$\geq 8 \mathrm{~h}$ & $3,902(32.4)$ & $5,095(31.5)$ & 43.4 \\
\hline \multicolumn{4}{|l|}{ Depression } \\
\hline No & $20,217(77.6)$ & $24,162(68.8)$ & 45.6 \\
\hline Yes & $5,835(22.4)$ & $10,977(31.2)$ & 34.7 \\
\hline \multicolumn{4}{|l|}{$B M I\left(k g ~ m^{-2}\right)$} \\
\hline $\begin{array}{l}\leq 18.5 \\
\text { (Underweight) }\end{array}$ & $609(1.8)$ & $947(2.1)$ & 39.1 \\
\hline 18.5-25 (Normal) & $14,561(42.9)$ & $19,261(41.8)$ & 43.1 \\
\hline $\begin{array}{l}25-30 \\
\text { (Overweight) }\end{array}$ & $12,803(35.6)$ & $15,440(33.5)$ & 45.3 \\
\hline$>30$ (Obese) & 6,677 (19.7) & $10,464(22.7)$ & 39.0 \\
\hline
\end{tabular}

$\left(\mathrm{OR}=0.64, P=1.1 \times 10^{-128}\right.$, Supplementary Table 11). Morning persons are less prevalent in extreme BMI groups, namely the underweight $(\leq 18.5)$ and the obese $(\geq 30)$ group (Table 1 , Supplementary Fig. 2). However, we found that after for adjusting for age and sex, the prevalence of morning persons decreases monotonically across increasing BMI categories (Supplementary Table 11). 


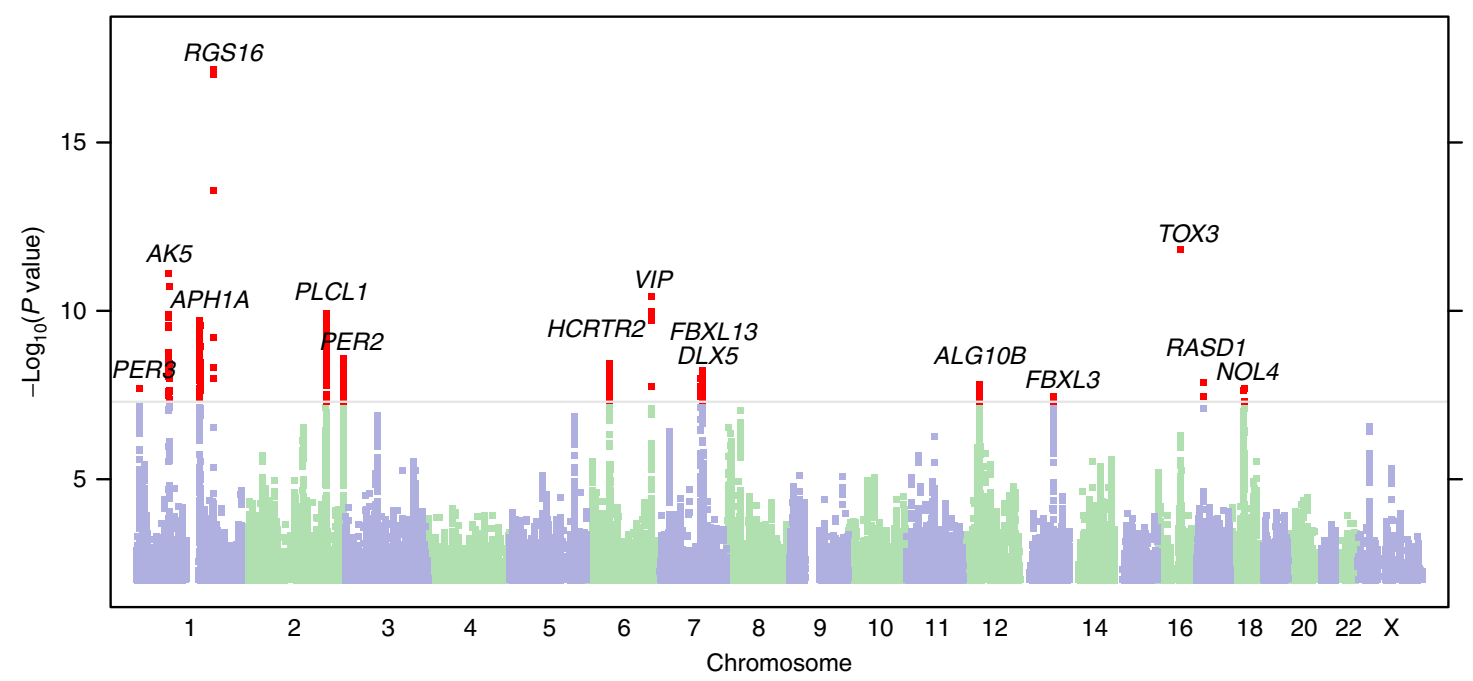

Figure 1 | Manhattan plot of the GWAS of being a morning person. The grey line corresponds to $P=5.0 \times 10^{-8}$, and the results above this threshold are shown in red. Gene labels are annotated as the nearby genes to the significant SNPs.

We included age, sex and the first 5 PCs in a logistic regression model and computed likelihood ratio tests for association of each genotyped or imputed marker with morningness. Association test results were adjusted for a genomic inflation factor of 1.21 (Supplementary Data 1). For an equivalent study of 1,000 cases and 1,000 controls, the genomic inflation factor (known as $\lambda_{1,000}$ (ref. 16)) would be 1.005 . The Manhattan plot (Fig. 1) shows 15 morningness-associated regions with genome-wide significance $\left(P<5 \times 10^{-8}\right)$. Table 2 categorizes their index single nucleotide polymorphisms (SNPs) by nearby genes. We used Haploreg ${ }^{17}$, a web based computational tool to explore chromatin states, conservations and regulatory motif alterations using public databases, to understand the possible functional roles of these index SNPs (Supplementary Table 16 and Supplementary Data 2).

Genetic association analyses. Seven loci are near well-established circadian genes. $r$ s12736689 $\left(P=7.0 \times 10^{-18}\right)$ is in strong linkage disequilibrium (LD) $\left(r^{2}=0.89\right)$ with the nonsynonymous variant rs1144566 (H137R) of nearby gene RGS16 (Supplementary Fig. 3), a G protein signalling regulator that inactivates $\mathrm{G}$ protein alpha subunits. RGS16 knock-out mice were shown to have a longer circadian period ${ }^{18}$. rs9479402 $\left(P=3.9 \times 10^{-11}\right)$ is $54 \mathrm{~kb}$ upstream of VIP (Supplementary Fig. 4), a key neuropeptide in the SCN (ref. 19). Its intracerebroventricular administration was found to prolong rapid eye movement sleep in rabbits ${ }^{20}$. rs55694368 $\left(P=3.9 \times 10^{-11}\right)$ is $120 \mathrm{~kb}$ upstream of PER2 (Supplementary Fig. 5), which has been associated with human familial advanced sleep phase syndrome ${ }^{7}$. This SNP is located in a DNAse hypersensitive site (DHS) for five cell types, including pancreas adenocarcinoma, B-lymphocyte (GM12891 and GM12892), medulloblastoma and CD4 + cells (Supplementary Table 16B), and alters five regulatory motifs. (See details in Supplementary Tables 16 and 17). rs35833281 $\left(P=3.7 \times 10^{-9}\right)$ is $18 \mathrm{~kb}$ downstream of HCRTR2, or orexin receptor type 2 (Supplementary Fig. 6) and alters eight regulatory motifs (Supplementary Table 16). Mutations in HCRTR2 have been linked to narcolepsy in dogs and humans ${ }^{21,22}$. This SNP rs35833281 is in partial LD with two SNPs $\left(r^{2}=0.25\right.$ for $\mathrm{rs} 2653349$ and $r^{2}=0.31$ for rs3122169) on HCRTR2 that were suggested to associate with cluster headache and narcolepsy ${ }^{23}$. These SNPs were also but less significantly associated with morningness $\left(P=3.6 \times 10^{-7}\right.$ for rs2653349 and $P=1.8 \times 10^{-6}$ for rs3122169). rs11545787 $\left(P=1.4 \times 10^{-8}\right)$ is a $3^{\prime} \mathrm{UTR}$ variant of RASD1 (Supplementary Fig. 7), a $G$ protein signaling activator ${ }^{24}$ and is a promoter histone mark for six cell types (H1, umbilical vein endothelial, B-lymphocyte, lung fibroblasts, skeletal muscle myoblasts and epidermal keratinocyte), in a DHS for seven cell types (skeletal muscle myoblasts, fibroblast, hepatocytes, medulloblastoma, epidermal melanocytes, pancreatic islets and fibroblasts) (Supplementary Table 16). In fact, deletion of RASD1 has been shown to result in a reduction of photic entrainment in mouse ${ }^{25}$. rs11121022 $\left(P=2.0 \times 10^{-8}\right)$, known to alter three regulatory motifs, is $8 \mathrm{~kb}$ downstream of PER3 (Supplementary Fig. 8), which affects the sensitivity of the circadian system to light ${ }^{26}$ and is involved in sleep/wake activity ${ }^{27}$. Variation in PER3 has also been associated with delayed sleep syndrome and extreme diurnal preference $^{28}$. A recent smaller study ${ }^{13}$ identified another SNP (rs228697) as a significant association with diurnal preference; however, this SNP is much less significant in our GWAS $\left(P=5.3 \times 10^{-5}\right)$ and is in low LD with our index SNP rs11121022 $\left(r^{2}=0.08\right)$. rs9565309 $\left(P=3.5 \times 10^{-8}\right)$, locating in a DHS for 16 cell types (Supplementary Table 16, Supplementary Data 2), is an intronic variant of CLN5 and is $\sim 2 \mathrm{~kb}$ downstream of FBXL3 (Supplementary Fig. 9), part of the F-box protein family, which ubiquitinates light-sensitive cryptochrome proteins CRY1 and CRY2, and mediates their degradation ${ }^{29}$. Mutant FBXL3 mice were shown to have an extended circadian period $^{30}$.

We found four additional SNPs are linked to genes that are plausibly circadian by literature review for reported potential connections between the genes and circadian rhythms. rs1595824 $\left(P=1.2 \times 10^{-10}\right)$ is an intronic variant of PLCL1 (Supplementary Fig. 10), which is expressed predominantly in the central nervous system and binds to the $\gamma$-aminobutyric acid (GABA) type A receptor. $\operatorname{rs} 12965577\left(P=2.1 \times 10^{-8}\right)$ is an intronic variant of NOL4 (Supplementary Fig. 13), one of 20 genes with the most significant changes in expression in mice with a knockin mutation in the $\alpha 1$ subunit of the $\operatorname{GABA}(\mathrm{A})$ receptor $^{31}$. As most SCN neuropeptides are colocalized with GABA (ref. 32) and most SCN neurons have GABAergic synapses ${ }^{33}$, it is possible that PLCL1 and NOL4 have circadian roles. rs34714364 $(P=2.0 \times$ $\left.10^{-10}\right)$, an enhancer histone mark, known to alter 11 regulatory motifs, a synonymous variant of gene $C A 14$, is $3 \mathrm{~kb}$ away from APH1A (Supplementary Fig. 11). APH1A encodes a component 
Table 2 | Index significant SNPs that are associated with being a morning person.

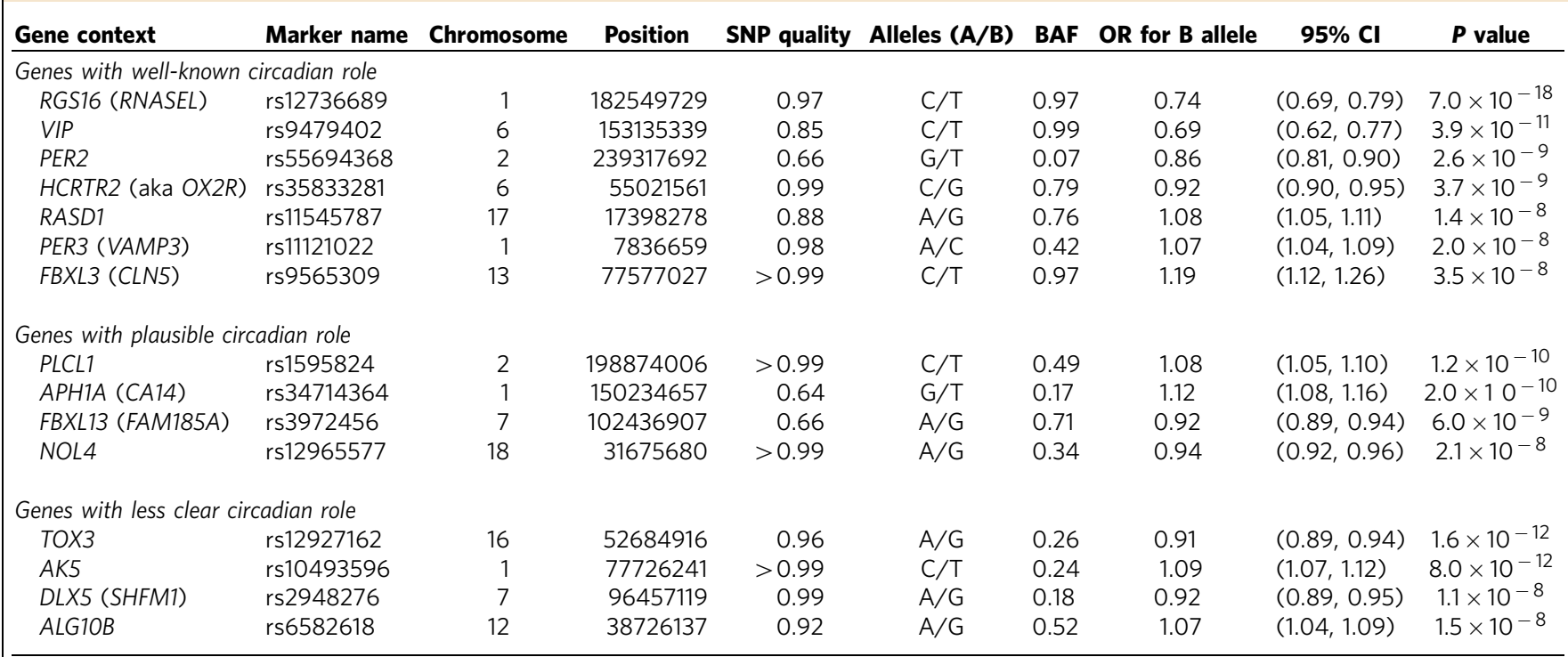

$\mathrm{BAF}, \mathrm{B}$ allele frequency; $\mathrm{Cl}$, confidence interval; SNP, single nucleotide polymorphism; gene context is the gene close to the index SNP; alleles $\mathrm{A}$ and $\mathrm{B}$ are assigned based on their alphabetical order; $\mathrm{OR}$, odds ratio for the $\mathrm{B}$ allele; $P$ values have been adjusted for a genomic control inflation factor of 1.21; position is the build hg19 map position of the SNP; SNP quality is $r^{2}$ from imputation.

of the $\gamma$-secretase complex which cleaves the $\beta$-amyloid precursor protein $^{34}$, and is regulated by orexin and the sleep-wake cycle ${ }^{35}$. This relationship of $\gamma$-secretase and sleep-wake cycle suggests a circadian role for $A P H 1 A$, but this region has many genes and further work is needed to verify this hypothesis. rs3972456 $\left(P=6.0 \times 10^{-9}\right)$, locating in a DHS for 8 cell types and known to alter three regulatory motifs, is an intronic variant of FAM185A and is $16 \mathrm{~kb}$ away from FBXL13 (Supplementary Fig. 12). FBXL13 also encodes a protein-ubiquitin ligase and may have a circadian role similar to FBXL3.

The relationship of the remaining loci to circadian rhythm is less clear. $\operatorname{rs} 12927162\left(P=1.6 \times 10^{-12}\right)$ is $104 \mathrm{~kb}$ upstream of TOX3 (Supplementary Fig. 14), a gene associated with restless leg syndrome ${ }^{36}$. The regional plot around rs12927162 shows that the next best SNP only has a $P$ value of $10^{-6}$. This SNP alters a POU2F2 motif, but we found no other functional annotation, and additional work is needed to verify this association. Notably, this SNP is not in $\operatorname{LD}\left(r^{2}=1.2 \times 10^{-4}\right)$ with the reported SNP rs3104767 for restless leg syndrome ${ }^{36}$ and SNPs rs3803662 and rs4784227 for breast cancer ${ }^{37,38}$ (Supplementary Table 12). And none of these SNPs have strong association with morningness $(P>0.01)$. rs $10493596\left(P=8.0 \times 10^{-12}\right)$ is $21 \mathrm{~kb}$ upstream of $A K 5$ (Supplementary Fig. 15), a gene that regulates adenine nucleotide metabolism expressed only in the brain ${ }^{39}$. rs2948276 $\left(P=1.1 \times 10^{-8}\right.$, Supplementary Fig. 16), known to locate in a DHS for three cell types and alter four motifs, is $192 \mathrm{~kb}$ downstream of DLX5 and $118 \mathrm{~kb}$ upstream of SHFM1, a region linked to split hand/foot malformation. rs6582618 $\left(P=1.5 \times 10^{-8}\right)$ is $2 \mathrm{~kb}$ upstream of $A L G 10 B$ (Supplementary Fig. 17), a gene with a role in regulation of cardiac rhythms ${ }^{40}$.

For the above significant loci, we performed stepwise conditional analyses to identify potential additional associated variants that are within $200 \mathrm{~kb}$ of the index SNPs. We iteratively added new SNPs into the model until no SNP had $P<1.0 \times 10^{-5}$. We identified one new SNP (Supplementary Table 6) respectively for the locus close to VIP (rs62436127, $\left.P=1.6 \times 10^{-6}\right), \quad A P H 1 A \quad\left(\mathrm{rs} 10888576, \quad P=5.0 \times 10^{-6}\right)$ and PER2 (rs114769095, $P=9.7 \times 10^{-6}$ ). Accounting for the $\sim 15,000$ total SNPs that we included in our conditional analysis, the secondary hit around VIP is significant $\left(P<3.3 \times 10^{-6}\right)$ but the other two are not.

We tested for interaction between these SNPs and age, gender, BMI, alcohol abuse, nicotine abuse and current caffeine use (see Supplementary Table 1 for definitions). First, we added each covariate into the null model of morning person versus age, sex and five PCs. Effects of BMI $\left(\mathrm{OR}=0.97 \mathrm{~kg}^{-1} \mathrm{~m}^{-2}, P=1.0 \times\right.$ $\left.10^{-125}\right)$ and nicotine abuse $\left(\mathrm{OR}=0.71, P=3.9 \times 10^{-41}\right)$ were significant (Supplementary Table 7A). We then added each SNP into each new null model. Effect sizes were not substantially altered, though $P$ values generally became less significant, consistent with the degree of reduction in sample size for these covariates (Supplementary Table 7B). We also added interaction terms (Supplementary Table 7C) for the significant SNPs and covariates to each model and found none that would be significant after accounting for multiple testing. In addition, we estimated SNP effects in three age groups $(<45,45-60$ and $>60)$ and found them consistent across these groups $(P>0.01$, Supplementary Table 7D). We also estimated $21 \%$ (95\% confidence interval (CI; 13\%, 29\%)) of the variance of the liability of morningness can be explained by genotyped SNPs, using Genome-wide Complex Trait Analysis (GCTA) (ref. 41) on a random subset of 10,000 samples due to computational constraints. Finally, we included the 'neutral' responders and defined a chronotype phenotype to describe morning, neutral and night person and then performed GWAS on it using a linear model with adjustment of age, sex and top five PCs. We found the results are largely similar to our morning-person GWAS. Detailed comparison (Supplementary Fig. 18) shows that in the chronotype GWAS the loci near FBXL3, RASD1 and NOL4 were no longer genome-wide significant. Two additional loci reached genome-wide significance at rs2975734 in MSRA (Supplementary Fig. 19) and rs9357620 in PHACTR1 (Supplementary Fig. 20, Supplementary Table 10). MSRA has been related to circadian rhythms in Drosophila ${ }^{42}$. PHACTR1 has not been reported to relate to circadian rhythms but has known associations with myocardial infarction ${ }^{43}$.

Pathway analyses. We used MAGENTA (ref. 44) to evaluate whether any biological pathways were enriched in our GWAS 
Table 3 | Top five morningness-associated pathways analysed by MAGENTA.

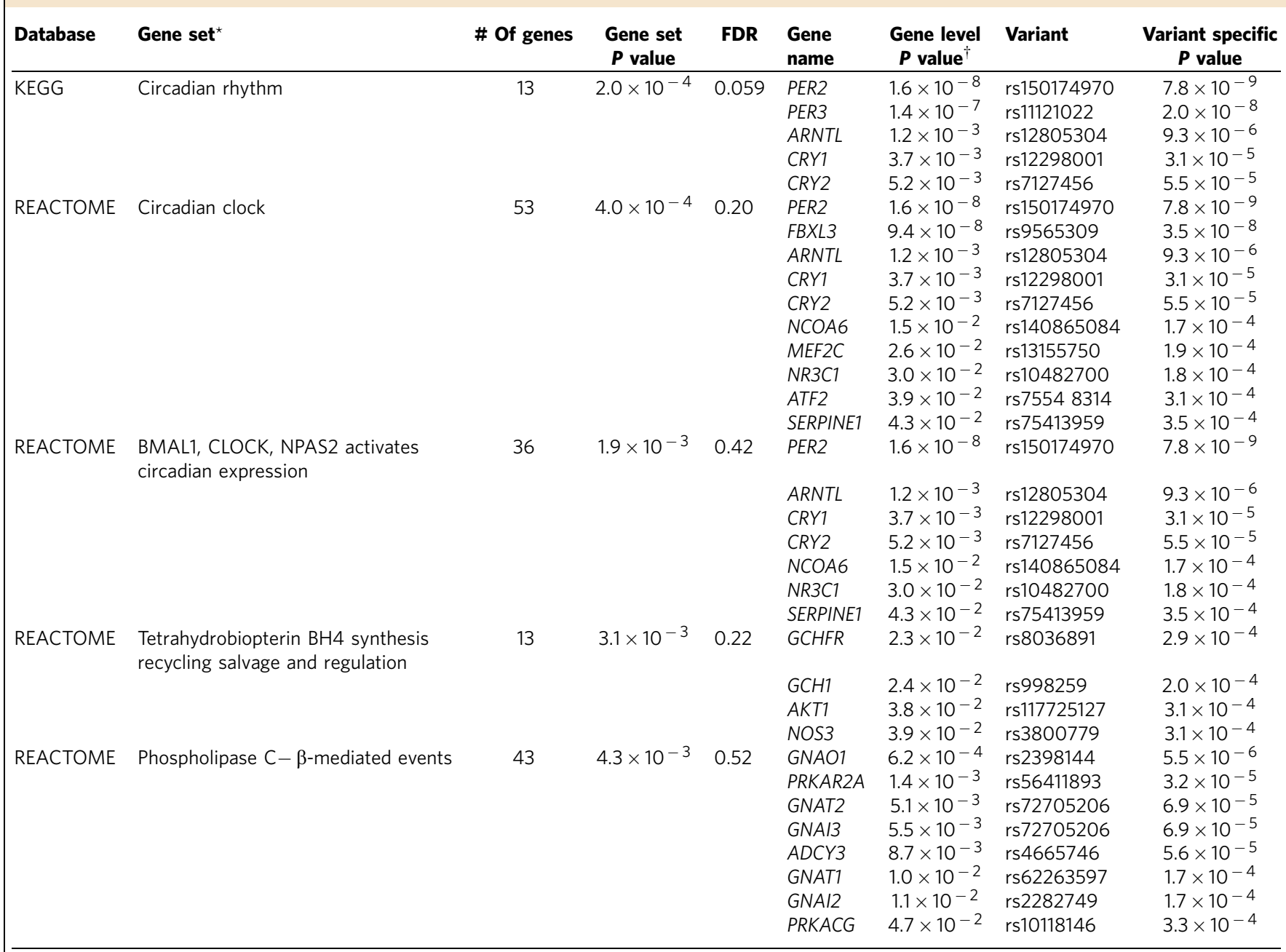

FDR, false discovery rate.

${ }^{*}$ The gene set is from the database of canonical pathways of 1,320 biologically defined gene sets (http://www.broadinstitute.org/gsea/msigdb/index.jsp).

${ }^{\dagger}$ For each pathway, we only include genes with a $P$ value $<0.05$.

results (Table 3 ). The top three pathways are circadian related and share four genes: $P E R 2$ (gene based $P$ value $=1.6 \times 10^{-8}$ ), ARNTL $\left(P=1.2 \times 10^{-3}\right)$, CRY1 $\left(P=3.7 \times 10^{-3}\right)$ and CRY2 $\left(P=5.2 \times 10^{-3}\right)$. In addition, PER3 $\left(P=1.4 \times 10^{-7}\right)$, in the KEGG circadian rhythm pathway, and FBXL3 $\left(P=9.4 \times 10^{-8}\right)$, in the REACTOME circadian clock pathway, have strong effects and were implicated in our GWAS. Other circadian genes also contribute to the enrichment of circadian pathways, but less significantly (Table 3). The $\mathrm{BH} 4$ related pathway (gene set $P=3.1 \times 10^{-3}$ ) has a major role in the biosynthesis of melatonin, serotonin and dopamine, which are important hormones involved in circadian rhythm regulation and brain function. The phospholipase C (PLC) $\beta$-mediated events pathway $(P=4.3$ $\left.\times 10^{-3}\right)$ includes GNAO1 $\left(P=6.2 \times 10^{-4}\right)$, GNAI3 $(P=5.5$ $\left.\times 10^{-3}\right)$, GNAT1 $\left(P=1.0 \times 10^{-2}\right)$ and many other $\mathrm{G}$ protein related genes involved in visual phototransduction. GNAT1 is related to night blindness ${ }^{45}$ and GNAI3 is known to interact with RGS16 (ref. 46). Interestingly, RGS16 is close to our GWAS top hit. This pathway also includes PRKAR2A $\left(P=1.4 \times 10^{-3}\right)$ and PRKACG $(P=0.047)$, which relate to cAMP dependent protein kinase $\mathrm{A}$, known to regulate critical processes in the circadian negative feedback loops ${ }^{47}$. Notably, except for the KEGG circadian rhythm pathway, which has a false discovery rate
0.06, all other associated pathways have false discovery rate $>0.2$, meaning the statistical evidence of the association is not strong.

We assessed correlations between morningness and related phenotypes with adjustment for potential confounders by regression with covariates for age, gender and ancestry (Table 4). The covariate-adjusted odds of having insomnia for morning people is $55 \%$ of that for night people $\left(P=1.5 \times 10^{-140}\right)$ and the adjusted odds of having sleep apnoea for morning people is $64 \%$ of that for night people $\left(P=4.0 \times 10^{-54}\right)$. Morning people are also less likely to require $>8 \mathrm{~h}$ of sleep $\left(\mathrm{OR}=0.69, P=6.3 \times 10^{-53}\right)$, to sleep soundly $\left(\mathrm{OR}=0.81, P=6.8 \times 10^{-24}\right)$, to have restless leg syndrome $\left(\mathrm{OR}=0.71, P=4.1 \times 10^{-15}\right)$ and sweat while sleeping $\left(\mathrm{OR}=0.90, \quad P=7.9 \times 10^{-6}\right)$ after adjusting for covariates. Sleepwalking and actual amount of sleep do not correlate with morningness $(P>0.1)$ in the full model. These associations are consistent with previous studies of insomnia ${ }^{48}$, sleep apnoea ${ }^{49}$ and sleep needed ${ }^{50}$. We calculated the association between the 15 GWAS identified SNPs and these eight sleep phenotypes (Supplementary Table 4) but found no significant associations. In addition, we looked up the SNPs and their proxies in the latest BMI GWAS from the GIANT consortium ${ }^{51}$ and the latest major depressive disorder GWAS from the CONVERGE Consortium ${ }^{52}$. 
Table 4 | Association of morningness and other phenotypes adjusting for age, sex, and 5 PC.

\begin{tabular}{|c|c|c|c|c|}
\hline Other phenotype & Sample size & Effect size $^{\star}$ & $95 \% \mathrm{Cl}$ & $P$ value \\
\hline \multicolumn{5}{|c|}{ Model: logistic regression of the binary phenotype versus morning person, age, sex and top five $P C s$} \\
\hline Insomnia & 40,976 & $\mathrm{OR}=0.41$ & $(0.39,0.42)$ & $<1.0 \times 10^{-200}$ \\
\hline Sleep apnea & 60,184 & $\mathrm{OR}=0.64$ & $(0.61,0.68)$ & $4.0 \times 10^{-54}$ \\
\hline Sleep needed $(\geq 8 \mathrm{~h})$ & 32,114 & $\mathrm{OR}=0.69$ & $(0.66,0.72)$ & $6.3 \times 10^{-53}$ \\
\hline Sound sleeper & 41,755 & $\mathrm{OR}=0.81$ & $(0.78,0.84)$ & $6.8 \times 10^{-24}$ \\
\hline Restless leg syndrome & 30,954 & $\mathrm{OR}=0.71$ & $(0.65,0.77)$ & $4.1 \times 10^{-15}$ \\
\hline Sweat while sleeping & 41,393 & $\mathrm{OR}=0.90$ & $(0.86,0.94)$ & $7.9 \times 10^{-6}$ \\
\hline Sleep walk & 31,533 & $\mathrm{OR}=1.05$ & $(0.97,1.15)$ & 0.24 \\
\hline Average daily sleep duration ( $\geq 8 \mathrm{~h}$ ) & 28,245 & $\mathrm{OR}=0.96$ & $(0.91,1.01)$ & 0.11 \\
\hline Depression & 61,191 & $\mathrm{OR}=0.61$ & $(0.59,0.63)$ & $3.5 \times 10^{-138}$ \\
\hline \multicolumn{5}{|c|}{ Model: linear regression of the continuous phenotype versus morning person, age, sex and top five PCs } \\
\hline BMI $\left(\mathrm{kg} \mathrm{m}^{-2}\right)$ & 80,042 & Slope $=-0.99\left(\mathrm{~kg} \mathrm{~m}^{-2}\right)$ & $(-1.07,-0.91)$ & $1.6 \times 10^{-125}$ \\
\hline
\end{tabular}

$\mathrm{BMI}$, body mass index; $\mathrm{Cl}$, confidence interval; $\mathrm{OR}$, odds ratio; $\mathrm{PC}$, principal component.

*In logistic regressions, the effect size is OR that describes the ratio of the odds of answering 'yes' to binary phenotypes in morning persons to the odds of answering 'yes' to binary phenotypes in night persons. In linear regression, the slope describes the difference of the average value (for example, BMI) in the morning persons and that in the night persons.

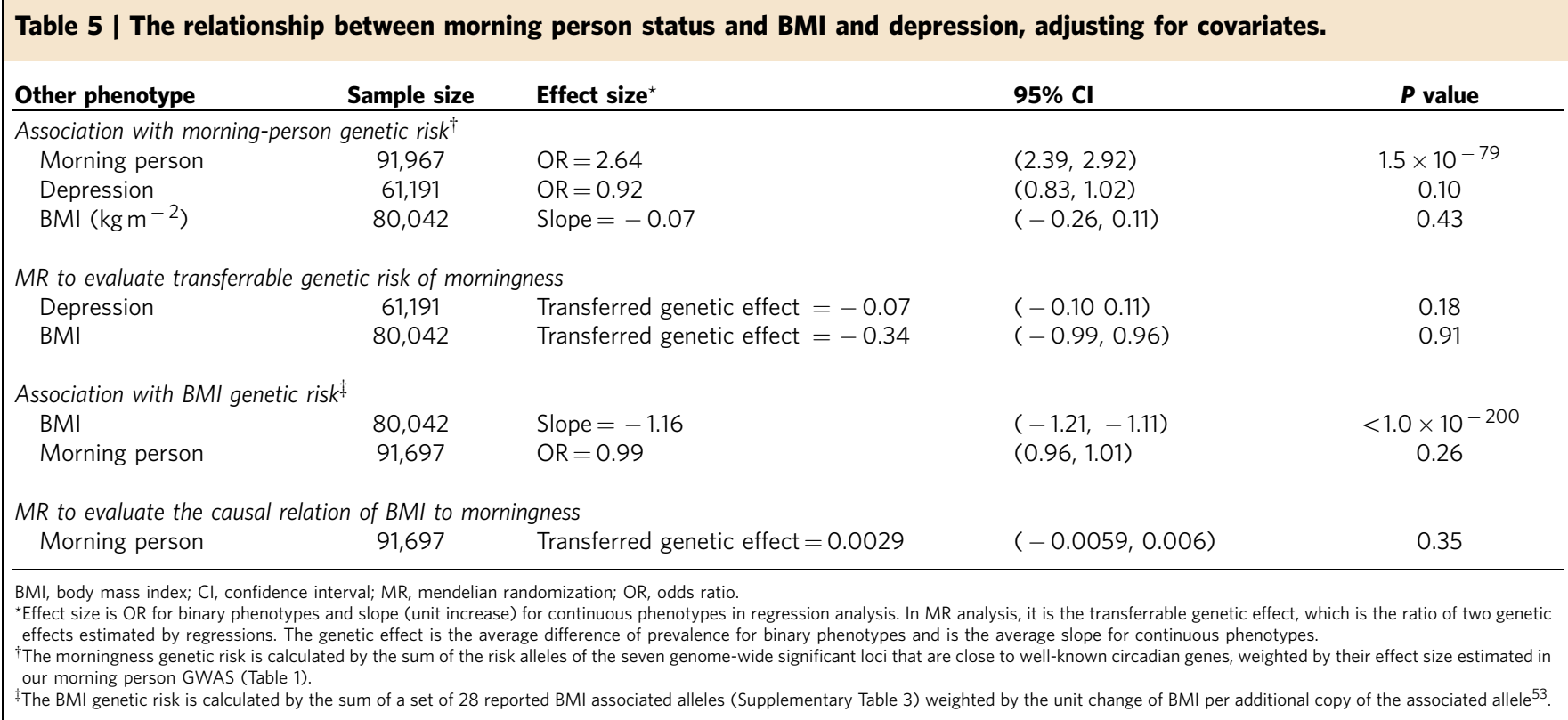

But we did not find significant associations (Supplementary Table 4B,C).

We examined previously identified associations of morningness with BMI (ref. 5) and depression ${ }^{6}$. We found that that the covariate-adjusted odds for morning people to report depression is $61 \%$ of that for night people $\left(P=3.5 \times 10^{-138}\right)$, and the average BMI for morning people is $0.99 \mathrm{~kg} \mathrm{~m}^{-2}$ lower $\left(P=1.6 \times 10^{-125}\right)$, adjusting for covariates (Table 4$)$. We also calculated the association between the 15 significant GWAS SNPs and depression and BMI but found no significant associations (Supplementary Table 4).

MR analyses. We used a MR approach to find evidence in support of a causal relationship of morningness with BMI. We first calculated a morningness genetic risk score by summing the risk alleles of the seven circadian related SNPs weighted by their effects, then regressed morningness or BMI against this instrument variable while adjusting for covariates (age, sex and top five $\mathrm{PCs}$ ), and consequently estimated the ratio of the covariate- adjusted genetic effect for morningness to that for BMI (Methods section). Morningness is highly correlated (F statistic $=19.0$, $P=2.1 \times 10^{-80}$ in the linear regression model and $P=1.5 \times$ $10^{-79}$ in the logistic regression) with the genetic risk, but BMI $(P=0.43)$ is not (Table 5). We further estimated the transferred genetic effect, that is, the effect from genetically elevated chance of being a morning person on BMI as $-0.34 \mathrm{~kg} \mathrm{~m}^{-2}(95 \% \mathrm{CI}$ : $(-0.99,0.96), P=0.91)$ per unit increase of probability of being a morning person. Similarly, we found that depression is not significantly correlated with morningness genetic risk $(P=0.10)$. We estimated a non-significant transferred genetic effect of morningness on depression: the probability of depression decreases by 0.07 (95\% CI: $(-0.10,0.11), P=0.18)$ per unit increase of probability of being a morning person. Thus, we did not find evidence for morningness to be protective of depression or high BMI. Notably, the power of the MR analysis is governed by the strength of the correlation between morningness and its genetic risk as well as the magnitude of the transferred genetic effect of morningness on BMI or depression. We ran simulations (Methods section) to assess the power for our MR and found that 
our current sample sizes, though large by conventional standards, only lead to moderate power in our MR analysis of morningness and BMI and depression (Supplementary Table 8). If the observed correlation is entirely causal, our analysis has only $\sim 40 \%$ power. Our reported lack of statistical evidence in our MR analysis could be due to constrained study power.

We also conducted an MR analysis of BMI on morningness. We retrieved the morningness GWAS results for a set of 28 previously reported BMI associated SNPs (ref. 53) and found rs1558902, an intronic variant of FTO, had some evidence for association with morningness $\left(P=6.0 \times 10^{-6}\right.$, Supplementary Table 5). We then calculated a BMI genetic risk with this set of SNPs using the previously reported effect sizes. It is highly correlated with BMI (F statistic $=47.4, P<1.0 \times 10^{-200}$ ) but we found it to be uncorrelated with morningness $(P=0.26)$ and found no support for a causal relationship (transferred genetic effect $=0.0029,95 \%$ CI: $(-0.0059,0.006), P=0.35)$. Our power calculation (Supplementary Table 8 ) shows that this MR analysis is well powered $(\sim 80 \%)$ to show evidence of a causal relationship between BMI and morningness, assuming the observed correlation is entirely causal.

\section{Discussion}

We identified many loci significantly associated with morningness but were unable to find clear genetic associations in our GWAS analysis of related sleep phenotypes, such as insomnia, sleep apnoea, sleep needed, sleeping soundly and sweating while sleeping. These sleep phenotypes may be more genetically heterogeneous and our current sample sizes, while large by most standards, maybe still be too small for discoveries. It is also possible that environmental factors mediate the association between morningness and these sleep phenotypes. These other phenotypes may also be more subject to possible self-reporting bias. We assessed morningness with simple questions and did not consider light exposure, season, geography and other factors, it is possible that better results would be obtained from using moredetailed surveys (such as the standard Horne-Ostberg questionnaire $^{2}$ ). We have also considered the effect of smoking, drinking and caffeine consumption in our analysis but with limited thoroughness for these phenotypes. More-detailed phenotyping would be desirable for future studies, though GWAS typically do not adjust for such factors. An analysis including more refined estimates of these covariates would yield more accurate estimates of effect sizes and could reveal information about mechanism, if some associations with sleep phenotypes are mediated by these other behaviours.

For known circadian genes such as DEC1, DEC2, BMAL1, CRY1 and CRY2, we did not find signals that were genome-wide significant. Specifically, within $100 \mathrm{~kb}$ windows of each gene, we had 1,712 SNPs for DEC1 with a minimum $P$ value 0.01 ; we had 689 SNPs for DEC2 with a minimum $P$ value $7.8 \times 10^{-4}$; we had 835 SNPs for $B M A L 1$ and a minimum $P$ value of $1.0 \times 10^{-6}$; we had 804 SNPs for $C R Y 1$ and a minimum $P$ value of $4.4 \times 10^{-6}$; we have 504 SNPs for $C R Y 2$ and a minimum $P$ value of $8.9 \times 10^{-6}$. Some of these genes may have a less important role in morningness, or may not have genetic variation that could be identified by GWAS. However, the associations in BMAL1, CRY1 and CRY2 are suggestive and additional data may confirm signals in these genes.

Another large-scale genetic study of chronotype ${ }^{54}$ using the UK Biobank has recently been completed, with results largely consistent with our own. Specifically, that study reports genomewide-significant loci at RGS16, AK5, PER2 and HCRTR2, as well as near FBXL13 and APH1A. Further work will be needed to assess replication of other loci not genome-wide significant in both studies.
Our MR analysis did not provide evidence for a causal relationship of morningness on BMI or depression. We have checked MR assumptions (Supplementary Tables 13-15). The F statistics is 19.0 in the linear regression of morning person. Since morningness is binary, we calculated a generalized coefficient of determination for logistic regression: Nagelkerke's $R^{2}=0.0056$. Without direct translation between the $R^{2}$ and the F statistics, we assumed that this small scale of $R^{2}$ could indicate our instrument is weak and our MR analysis could be underpowered. We verified that PCs are associated with both risk and outcomes (Supplementary Table 13A), so we have adjusted for them in our MR analysis (Supplementary Table 13A). In addition, we found the preference of sweet foods (effect $=0.147, P=7.1 \times 10^{-3}$, Supplementary Table 14) is moderately associated with morningperson genetic risk $\left(\mathrm{OR}=0.15, P=7.1 \times 10^{-3}\right)$ and $\mathrm{BMI}(\mathrm{OR}=$ $1.009 \mathrm{~kg}^{-1} \mathrm{~m}^{-2}, P=1.5 \times 10^{-10}$, Supplementary Table $\left.14 \mathrm{~A}\right)$ and depression $\left(\mathrm{OR}=1.24, P=6.9 \times 10^{-27}\right)$. We hence included the preference of sweet foods in our MR analysis but found no changes in our conclusion $(P>0.01)$.

In addition, we also checked for assumptions in our MR analysis of BMI on morningness (Supplementary Tables 13-15). We found PCs are associated with BMI risk and morning person (Supplementary Table 13B). We also identified current caffeine use (Supplementary Table 14B) is associated with BMI genetic risk (effect $=10.5 \mathrm{~kg}^{-1} \mathrm{~m}^{-2}, \quad P=1.3 \times 10^{-6}$ ) and morning person $\left(\right.$ effect $\left.=18.2, P=4.5 \times 10^{-11}\right)$. Adjusting for PCs and current caffeine use did not lead to change to our result (Supplementary Table 15B). Our MR analysis could not rule out canalization or developmental compensation, by which individuals adapt in response to genetic change in a way that the expected effect of the change is reduced ${ }^{55}$. Our analysis also did not test for non-linear relationships between the phenotypes.

Among BMI risk SNPs, we found an FTO variant strongly correlated with morningness (Supplementary Table 5). Our MR analysis using a BMI genetic risk score as an instrument variable did not find evidence to support a more general effect of BMI genetic risk on morningness. There may be pleiotropy specifically at the FTO locus, instead of a more general casual effect of BMI. Moreover, their strong association may reflect effects of other factors, such as environment, socioeconomics, personality or other genetic variables through independent mechanisms.

\section{Methods}

23andMe cohort. Participants in the 23andMe cohort were customers of 23andMe, Inc., a personal genetics company, who had been genotyped as part of the 23andMe Personal Genome Services. DNA extraction and genotyping were performed on saliva samples by the National Genetics Institute (NGI), a Clinical Laboratory Improvement Amendments (CLIA)-certified clinical laboratory and subsidiary of the Laboratory Corporation of America. Samples were genotyped on one of two platforms. About $35 \%$ of the participants were genotyped on the Illumina HumanHap550 + BeadChip platform, which included SNPs from the standard HumanHap550 panel augmented with a custom set of $\sim 25,000$ SNP selected by 23 andMe. Two slightly different versions of this platform were used, as previously described ${ }^{14}$. The remaining $65 \%$ of participants were genotyped on the Illumina HumanOmniExpress + BeadChip. This platform has a base set of 730,000 SNPs augmented with $\sim 250,000$ SNPs to obtain a superset of the HumanHap550 + content, as well as a custom set of $\sim 30,000$ SNPs. Every sample that did not reach a $98.5 \%$ call rate for SNPs on the standard platforms was reanalyzed. Individuals whose analyses repeatedly failed were contacted by 23 andMe customer service to provide additional samples.

We collected phenotypes by inviting participants to login in www.23andme.com to answer surveys that are either comprehensive ones with multiple questions on a subject matter or quick questions. We defined phenotypes by combining the answers to questions on the same subject. For example, as shown in Supplementary Table 2, our morning person phenotype definition is from combining the answers to two questions that asking if the participant is naturally a night person or morning person. For each question, we classify the answer as night person, morning person or missing. Then if one answer is missing, we use the other answer as the phenotype value, and if one answer is morning person but the other is night person, we treated the phenotype value as missing. Similarly, we used appropriate 
combination rules to derive other phenotypes from multiple survey questions (see more in Supplementary Table 2).

The study protocol and consent form were approved by the external Association for the Accreditation of Human Research Protection Programsaccredited Institutional Review Board, Ethical \& Independent Review Services. For a small number of participants $(n=167)$ under the age of 18 years, consent was provided by a parent, guardian or legally authorized adult.

GWAS analysis for 23andMe European samples. For our standard GWAS, we restricted participants to a set of individuals who have >97\% European ancestry, as determined through an analysis of local ancestry via comparison to the three HapMap 2 populations ${ }^{56}$. A maximal set of unrelated individuals was chosen for the analysis using a segmental identity-by-descent estimation algorithm ${ }^{57}$. Individuals were defined as related if they shared $>700 \mathrm{cM}$ identity-by-descent, including regions where the two individuals share either one or both genomic segments identical-by-descent. This level of relatedness (roughly $20 \%$ of the genome) corresponds approximately to the minimal expected sharing between first cousins in an outbred population.

Participant genotype data were imputed against the August 2010 release of 1,000 Genomes reference haplotypes ${ }^{58}$. First, we used Beagle ${ }^{59}$ (version 3.3.1) to phase batches of 8,000-9,000 individuals across chromosomal segments of no $>10,000$ genotyped SNPs, with overlaps of 200 SNPs. We excluded SNPs with minor allele frequency $<0.001$, Hardy-Weinberg equilibrium $P<10^{-20}$, call rate $<95 \%$, or with large allele frequency discrepancies compared with the 1,000 Genomes reference data. We identified the discrepancies by computing a $2 \times 2$ table of allele counts for the European 1,000 Genomes samples and 2,000 randomly sampled 23andMe customers with European ancestry and excluded SNPs with $\chi^{2}$ test $P$ value $<10^{-15}$. We then assembled full-phased chromosomes by matching the phase of haplotypes across the overlapping segments. We imputed each batch against the European subset of 1,000 Genomes haplotypes using Minimac $(2011-10-27)^{60}$, using five rounds and 200 states for parameter estimation.

For the non-pseudoautosomal region of the $\mathrm{X}$ chromosome, males and females were phased together in segments, treating the males as already phased; the pseudoautosomal regions were phased separately. We assembled fully phased X chromosomes, representing males as homozygous pseudo-diploids for the nonpseudoautosomal region. We then imputed males and females together using Minimac as with the autosomes.

For morning and night person comparisons, we computed association test results by logistic regression assuming additive allelic effects. For tests using imputed data, we used the imputed dosages rather than best-guess genotypes. We used covariates age, gender, and the top five PC to account for residual population structure. The GWAS association test $P$ values were computed using a likelihood ratio test. Results for the $\mathrm{X}$ chromosome are computed similarly, with men coded as if they were homozygous diploid for the observed allele.

Imputed results were computed for 7,381,496 SNPs having an average imputation $r^{2}>0.5$ and a minimum within-batch $r^{2}>0.3$, and removing SNPs with evidence of a strong batch effect $\left(P<10^{-50}\right)$, measured by ANOVA of dosages versus batches. For genotyped SNPs, we identified 854,959 SNPs with a minor allele frequency $>0.1 \%$, call rate $>90 \%$, Hardy-Weinberg $P>10^{-20}$ in European 23andMe participants and $P>10^{-50}$ for an effect of genotyping date on allele frequency. To create a single merged result set, for 806,041 SNPs with both imputed and genotyped results passing these quality filters, we selected the imputed result. After applying these filters and removing a small number of results that did not converge, we were left with association test results for 7,427,422 SNPs.

Pathway analysis of morningness. We first downloaded a database of canonical pathways of 1,320 biologically defined gene sets ${ }^{61}$, then used gene set enrichment analysis ${ }^{61}$, implemented in MAGENTA (ref. 44), on our morningness GWAS results. MAGENTA first assigns SNPs to a gene within $110 \mathrm{~kb}$ upstream and $40 \mathrm{~kb}$ downstream of transcript boundaries. The most significant SNP in this gene is then adjusted for confounders (gene size, SNP density and LD) in a regression framework to obtain a score for each gene. Then genes are then ranked according to their scores and then a gene set enrichment analysis-based approach was used to test whether predefined sets of functionally related genes are enriched for genes associated with morning person, more than would be expected by chance. MAGENTA counts the number of genes (enrichment score) with scores ranking above the 95th percentile. To evaluate the significance of each pathway, MAGENTA randomly sample 10,000 gene sets from the genome that are of identical size to the pathway and compare the observed enrichment score to the resampled enrichment score. To adjust for multiple testing, it estimates the false discovery rate by comparing the observed normalized enrichment score to all resampled normalized enrichment score.

Relationship of morningness and other phenotypes. For binary phenotypes such as insomnia, sleep apnoea, we used a logistic regression to estimate the effect of morningness after adjusting for age, sex and top five PCs. For the continuous phenotype BMI, we used a linear regression model instead. Morningness is part of sleep and its aetiology intertwines with other sleep phenotypes, so it is difficult to dissect the causal relationship. But BMI and depression are not directly involved in sleep, we can treat the genetic risk of being a morning person as an instrument variable for causal inference. We calculated a morningness genetic risk by averaging the genotypes of the seven SNPs that are close to known circadian genes weighted by their log odds ratio. Then we carried out a MR analysis to evaluate the causal role of morningness. The transferred genetic effect on morningness is estimated by dividing the genetic risk effect of the phenotype to that of the morning-person phenotype. For a continuous phenotype (for example, BMI), this genetic risk effect is the change of mean per unit increase of risk estimated by a linear regression. For a binary phenotype (for example, depression and morningness), this genetic risk effect is the change of probability per unit increase of risk estimated by a logistic regression model ${ }^{62,63}$. The $95 \%$ CI of the transferred genetic effect is estimated using the Bootstrap, where we resampled the cohort for 1,000 times to obtain resampled transferred genetic effects. We then calculated two-sided $P$ value by comparing the observed transferred genetic effect and the resampled transferred genetic effects.

For the analysis of the transferred genetic effect of BMI on morningness, we calculated a BMI risk by averaging the genotypes of a total of 28 previously reported BMI loci weighted by their reported effect sizes ${ }^{53}$. We then estimated the transferred genetic effect as the ratio of the genetic risk effect on morningness to that on BMI. The confidence interval and statistical inference is done using Bootstrapping.

In addition, we performed simulations to evaluate the power of our MR analysis ${ }^{64}$. For the analysis of morningness to depression, we kept the genetic risk, age, sex, top five PCs and morning person as observed, and then simulated depression from a Bernoulli distribution with expectation calculated from a logistic regression model. In that model, we included morningness, age, sex and top five PCs as predictors. Their effects were estimated by regressing our observed depression phenotypes against these predictors, except that for morningness, we specified its transferred genetic effect using hypothetical values. Similarly, we simulated BMI using a linear regression with predictors as morningness and other covariates, with the effect of covariates estimated from our data and the transferred genetic effect of morningness hypothetically chosen. The simulation to evaluate the causal role of BMI on morningness was conducted in a similar fashion.

\section{References}

1. Kleitman, N. Sleep and Wakefulness as Alternating Phases in the Cycle of Existence. xii 638 p.Univ. of Chicago, 1939).

2. Horne, J. A. \& Ostberg, O. A self-assessment questionnaire to determine morningness-eveningness in human circadian rhythms. Int. J. Chronobiol. 4, 97-110 (1976).

3. Welsh, D. K., Takahashi, J. S. \& Kay, S. A. Suprachiasmatic nucleus: cell autonomy and network properties. Annu. Rev. Physiol. 72, 551-577 (2010).

4. Zisapel, N. Circadian rhythm sleep disorders: pathophysiology and potential approaches to management. CNS Drugs 15, 311-328 (2001).

5. Roenneberg, T., Allebrandt, K. V., Merrow, M. \& Vetter, C. Social jetlag and obesity. Curr. Biol. 22, 939-943 (2012)

6. Germain, A. \& Kupfer, D. J. Circadian rhythm disturbances in depression. Hum. Psychopharmacol. 23, 571-585 (2008).

7. Toh, K. L. et al. An hPer2 phosphorylation site mutation in familial advanced sleep phase syndrome. Science 291, 1040-1043 (2001).

8. Takano, A. et al. A missense variation in human casein kinase I epsilon gene that induces functional alteration and shows an inverse association with circadian rhythm sleep disorders. Neuropsychopharmacology 29, 1901-1909 (2004).

9. Pereira, D. S. et al. Association of the length polymorphism in the human Per3 gene with the delayed sleep-phase syndrome: does latitude have an influence upon it? Sleep 28, 29-32 (2005).

10. Allebrandt, K. V. \& Roenneberg, T. The search for circadian clock components in humans: new perspectives for association studies. Braz. J. Med. Biol. Res. 41 716-721 (2008).

11. Raizen, D. M. \& Wu, M. N. Genome-wide association studies of sleep disorders. Chest 139, 446-452 (2011).

12. Gottlieb, D. J. et al. Novel loci associated with usual sleep duration: the CHARGE Consortium Genome-Wide Association Study. Mol. Psychiatry 20, 1232-1239, doi:10.1038/mp.2014.133 (2014).

13. Hida, A. et al. Screening of clock gene polymorphisms demonstrates association of a PER3 polymorphism with morningness-eveningness preference and circadian rhythm sleep disorder. Sci. Rep. 4, 6309 (2014).

14. Eriksson, N. et al. Web-based, participant-driven studies yield novel genetic associations for common traits. PLoS Genet. 6, e1000993 (2010).

15. Carrier, J., Monk, T. H., Buysse, D. J. \& Kupfer, D. J. Sleep and morningnesseveningness in the 'middle' years of life (20-59 y). J. Sleep Res. 6, 230-237 (1997).

16. Freedman, M. L. et al. Assessing the impact of population stratification on genetic association studies. Nat. Genet. 36, 388-393 (2004).

17. Ward, L. D. \& Kellis, M. HaploReg: A resource for exploring chromatin states, conservation, and regulatory motif alterations within sets of genetically linked variants. Nucleic Acids Res. 40, D930-D934 (2012). 
18. Doi, M. et al. Circadian regulation of intracellular G-protein signalling mediates intercellular synchrony and rhythmicity in the suprachiasmatic nucleus. Nat. Commun. 2, 327 (2011).

19. Hastings, M. H., Brancaccio, M. \& Maywood, E. S. Circadian Pacemaking in cells and circuits of the suprachiasmatic nucleus. J. Neuroendocrinol. 26, 2-10 (2013).

20. Obal, Jr. F., Opp, M., Cady, A. B., Johannsen, L. \& Krueger, J. M. Prolactin, vasoactive intestinal peptide, and peptide histidine methionine elicit selective increases in REM sleep in rabbits. Brain Res. 490, 292-300 (1989).

21. Mignot, E. et al. Genetic linkage of autosomal recessive canine narcolepsy with a mu immunoglobulin heavy-chain switch-like segment. Proc. Natl Acad. Sci. USA 88, 3475-3478 (1991).

22. Lin, L. et al. The sleep disorder canine narcolepsy is caused by a mutation in the hypocretin (orexin) receptor 2 gene. Cell 98, 365-376 (1999).

23. Rainero, I. et al. A polymorphism of the hypocretin receptor 2 gene is associated with cluster headache. Neurology 63, 1286-1288 (2004).

24. Fang, M. et al. Dexras1: a G protein specifically coupled to neuronal nitric oxide synthase via CAPON. Neuron 28, 183-193 (2000).

25. Cheng, H. Y. et al. Dexrasl potentiates photic and suppresses nonphotic responses of the circadian clock. Neuron 43, 715-728 (2004).

26. Van der Veen, D. R. \& Archer, S. N. Light-dependent behavioral phenotypes in PER3-deficient mice. J. Biol. Rhythms 25, 3-8 (2010).

27. Viola, A. U. et al. PER3 polymorphism predicts sleep structure and waking performance. Curr. Biol. 17, 613-618 (2007).

28. Archer, S. N. et al. A length polymorphism in the circadian clock gene Per3 is linked to delayed sleep phase syndrome and extreme diurnal preference. Sleep 26, 413-415 (2003).

29. Siepka, S. M. et al. Circadian mutant Overtime reveals F-box protein FBXL3 regulation of cryptochrome and period gene expression. Cell 129, 1011-1023 (2007).

30. Godinho, S. I. et al. The after-hours mutant reveals a role for Fbxl3 in determining mammalian circadian period. Science 316, 897-900 (2007).

31. Harris, R. A., Osterndorff-Kahanek, E., Ponomarev, I., Homanics, G. E. \& Blednov, Y. A. Testing the silence of mutations: transcriptomic and behavioral studies of GABA(A) receptor $\alpha 1$ and $\alpha 2$ subunit knock-in mice. Neurosci. Lett. 488, 31-35 (2011).

32. Moore, R. Y. \& Speh, J. C. GABA is the principal neurotransmitter of the circadian system. Neurosci. Lett. 150, 112-116 (1993).

33. Strecker, G. J., Wuarin, J. P. \& Dudek, F. E. GABAA-mediated local synaptic pathways connect neurons in the rat suprachiasmatic nucleus. J. Neurophysiol. 78, 2217-2220 (1997).

34. Francis, R. et al. aph-1 and pen-2 are required for Notch pathway signaling, $\gamma$-secretase cleavage of $\beta \mathrm{APP}$, and presenilin protein accumulation. Dev. Cell 3, 85-97 (2002).

35. Kang, J.-E. et al. Amyloid-beta dynamics are regulated by orexin and the sleep-wake cycle. Science 326, 1005-1007 (2009).

36. Winkelmann, J. et al. Genome-wide association study identifies novel restless legs syndrome susceptibility loci on 2p14 and 16q12.1. PLoS Genet. 7, e1002171 (2011).

37. Orr, N. et al. Genome-wide association study identifies a common variant in RAD51B associated with male breast cancer risk. Nat. Genet. 44, 1182-1184 (2012).

38. Cowper-Sal · lari, R. et al. Breast cancer risk-associated SNPs modulate the affinity of chromatin for FOXA1 and alter gene expression. Nat. Genet. 44, 1191-1198 (2012).

39. Noma, T. Dynamics of nucleotide metabolism as a supporter of life phenomena. J. Med. Invest. 52, 127-136 (2005).

40. Hayashi, K. et al. A KCR1 variant implicated in susceptibility to the long QT syndrome. J. Mol. Cell. Cardiol. 50, 50-57 (2011).

41. Yang, J., Lee, S. H., Goddard, M. E. \& Visscher, P. M. GCTA: A tool for genome-wide complex trait analysis. Am. J. Hum. Genet. 88, 76-82 (2011).

42. Wassef, R. et al. Methionine sulfoxide reductase A and a dietary supplement S-methyl-L-cysteine prevent Parkinson's-like symptoms. J. Neurosci. 27, 12808-12816 (2007)

43. Kathiresan, S. et al. Genome-wide association of early-onset myocardial infarction with single nucleotide polymorphisms and copy number variants. Nat. Genet. 41, 334-341 (2009).

44. Ayellet, V. S., Groop, L., Mootha, V. K., Daly, M. J. \& Altshuler, D. Common inherited variation in mitochondrial genes is not enriched for associations with type 2 diabetes or related glycemic traits. PLoS Genet. 6, e1001058, doi:10.1371/journal.pgen.1001058 (2010).

45. Dryja, T. P., Hahn, L. B., Reboul, T. \& Arnaud, B. Missense mutation in the gene encoding the alpha subunit of rod transducin in the Nougaret form of congenital stationary night blindness. Nat. Genet. 13, 358-360 (1996).

46. McDowall, M. D., Scott, M. S. \& Barton, G. J. PIPs: human protein-protein interaction prediction database. Nucleic Acids Res. 37, D651-D656 (2009)

47. Huang, G. et al. Protein kinase A and casein kinases mediate sequential phosphorylation events in the circadian negative feedback loop. Genes Dev. 21, 3283-3295 (2007).

48. Ong, J. C., Huang, J. S., Kuo, T. F. \& Manber, R. Characteristics of insomniacs with self-reported morning and evening chronotypes. J. Clin. Sleep Med. 3, 289-294 (2007)
49. Lucassen, E. A. et al. Evening chronotype is associated with changes in eating behavior, more sleep apnea, and increased stress hormones in short sleeping obese individuals. PLoS One 8, e56519 (2013).

50. Taillard, J., Philip, P. \& Bioulac, B. Morningness/eveningness and the need for sleep. J. Sleep Res. 8, 291-295 (1999).

51. Locke, A. E., Kahali, B. \& Sonja, B. I. Genetic studies of body mass index yield new insights for obesity biology. Nature 518, 197-206 (2015).

52. CONVERGE consortium. et al. Sparse whole-genome sequencing identifies to loci for major depressive disorder. Nature 523, 588-591 (2015).

53. Speliotes, E. K. et al. Association analyses of 249,796 individuals reveal 18 new loci associated with body mass index. Nat. Genet. 42, 937-948 (2010).

54. Jones, S. E. et al.The genetics of sleep patterns and their relationship with obesity and type 2 diabetes: A UK Biobank study http://dx.doi.org/10.1101/ 031369 (2015).

55. Waddington, C. H. Canalization of development and the inheritance of acquired characters. Nature 150, 563-565 (1942).

56. Falush, D., Stephens, M. \& Pritchard, J. K. Inference of population structure using multilocus genotype data: dominant markers and null alleles. Mol. Ecol. Notes 7, 574-578 (2007).

57. Henn, B. M. et al. Cryptic distant relatives are common in both isolated and cosmopolitan genetic samples. PLoS ONE 7, e34267, doi:10.1371/journal.pone. 0034267 (2012).

58. Altshuler, D., Lander, E. \& Ambrogio, L. A map of human genome variation from population scale sequencing. Nature 476, 1061-1073 (2010).

59. Browning, S. R. \& Browning, B. L. Rapid and accurate haplotype phasing and missing-data inference for whole-genome association studies by use of localized haplotype clustering. Am. J. Hum. Genet. 81, 1084-1097 (2007).

60. Howie, B., Fuchsberger, C., Stephens, M., Marchini, J. \& Abecasis, G. R. Fast and accurate genotype imputation in genome-wide association studies through pre-phasing. Nat. Genet. 44, 955-959 (2012).

61. Subramanian, A. et al. Gene set enrichment analysis: a knowledge-based approach for interpreting genome-wide expression profiles. Proc. Natl Acad. Sci. USA 102, 15545-15550 (2005).

62. Angrist, J. D., Imbens, G. W. \& Rubin, D. B. Identification of causal effects using instrumental variables. J. Am. Stat. Assoc. 91, 444-455 (1996).

63. Little, R. J., Long, Q. \& Lin, X. A comparison of methods for estimating the causal effect of a treatment in randomized clinical trials subject to noncompliance. Biometrics 65, 640-649 (2009).

64. Pierce, B. L., Ahsan, H. \& Vanderweele, T. J. Power and instrument strength requirements for Mendelian randomization studies using multiple genetic variants. Int. J. Epidemiol. 40, 740-752 (2011).

\section{Acknowledgements}

We thank the customers of 23andMe who answered surveys, Aaron Kleinman for discussion on MR, Brian Naughton, Emma Pierson, Cory McLean and Anna Guan for comments, as well as the employees of $23 \mathrm{andMe}$, who together made this research possible. Research reported in this publication was supported by the National Human Genome Research Institute of the National Institutes of Health under Award number R44HG006981. The content is solely the responsibility of the authors and does not necessarily represent the official views of the National Institutes of Health.

\section{Author contributions}

Y.H. and D.A.H. analysed the data and wrote the manuscript. A.S. performed the pathway analysis. D.T. helped interpret the results. N.E. developed analytical tools. J.Y.T. designed and supervised the study.

\section{Additional information}

Supplementary Information accompanies this paper at http://www.nature.com/ naturecommunications

Competing financial interests: All authors are current or former employees of and own stock or stock options in 23andMe, Inc.

Reprints and permission information is available online at http://npg.nature.com/ reprintsandpermissions/

How to cite this article: Hu, Y. et al. GWAS of 89,283 individuals identifies genetic variants associated with self-reporting of being a morning person. Nat. Commun. 7:10448 doi: $10.1038 /$ ncomms10448 (2016)

This work is licensed under a Creative Commons Attribution 4.0 International License. The images or other third party material in this article are included in the article's Creative Commons license, unless indicated otherwise in the credit line; if the material is not included under the Creative Commons license, users will need to obtain permission from the license holder to reproduce the material To view a copy of this license, visit http://creativecommons.org/licenses/by/4.0/ 